\title{
Culture and incentives conducive to quality and safety in health care
}

Compiled by Tom Smith

Please visit the QSHC website (http://www.qshc.com)

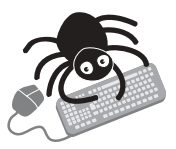
for links to these articles - many to full text.

The authors believe that, in the final analysis, major failures in health care are-more than anything else-a product of the distinctive culture of the organizations, the healthcare professions, and the health system.

A Walshe K, Shortell S. When things go wrong: how health care organizations deal with major failures. Health Affairs 2004;23:103-11.

Two main themes run through the papers in this issue of JournalScan, both of which seem central to new strategies to improve quality and safety in health care:

- an emphasis on culture relationships within organisations between managers and professionals, clinicians and patients; and

- incentives - ways of rewarding behaviour to enhance quality.

Learning from organisational failure A paper in Health Affairs begins by saying that, if healthcare organisations are to become exemplar in quality and safety, they should mimic the airline industry where "every airplane crash is carefully catalogued and painstakingly analysed to learn lessons for the future". If not, "important opportunities for improvement will be missed, and the chances are surely higher that similar failures will happen again". It examines examples of major failures from six countries-US, UK, Australia, New Zealand, Canada and the Netherlands - to explore how healthcare systems and organisations deal with these failures.

Analysis shows that common themes run through many of the instances of major failure:

- Longstanding problems which have been present and known about for years before they are brought to light.

- Well known but not handled.

- Lack of management systems: the organisations where these failures occur usually lack fundamental management systems for quality review, incident reporting, and performance management, or those systems have been bypassed with ease. They frequently show little collaboration between managers and clinicians and a lack of coherent clinical leadership. They are often isolated and inward looking organisations unwilling to learn from elsewhere.

"Perhaps the most important barrier to disclosure is the endemic culture of secrecy and protectionism in health care facilities in every country. Knowledge about these problems and responsibility for acting to tackle them are often fragmented across many people, who all know something about the problem or failure but don't necessarily know the full picture or have the authority or incentive to act. The capacity of individuals and organizations for selfdeception and post hoc rationalization in the face of unwelcome information often plays a part in their inaction."

The authors make four recommendations:

- "Action is needed to make the systems for identifying and highlighting failures work more effectively, mainly by creating strong incentives to report and by removing disincentives and barriers to reporting."

- "It is also evident that in most cases of major failure the systems for quality management ... are unable to cope with the problems."

- "Investigations of major failures ... appear ... chaotic and uncertain". A more comprehensive approach to quality is needed.

- "There should also be explicit mechanisms for ensuring that the lessons from major failures are translated into explicit and agreed recommendations for changes in practice which are then implemented."
Learning how to adopt best practice in safety from others - In America the Joint Commission on Accreditation of Healthcare Organisations 2 years ago updated its requirements, "emphasising organizational responsibility for patient safety". Its additional standards require leaders of organisations to "create a culture of safety, implement patient safety programs, ensure adequate and timely responses to adverse events when they occur ... and prevent accidental harm through the prospective analysis".

"Most of these recommended policies and practices are based on well-established research evidence or expert consensus [but] few, if any, are widely adopted. The dawning realization is that, while best practices are intended to apply to all situations and environments, contextualisation is necessary to implement them effectively."

The paper is concerned with how Canadian hospitals might learn about best practice transfer and the authors consider five main influencing factors that affect the ease and efficacy of knowledge transfer.

(1) Environmental characteristics and interorganisational knowledge transfer

An organisation's environment includes other organisations and these can influence learning in a focal unit in two ways: (a) by prompting arms' length imitation and (b) by contact learning. The latter is argued to be "less susceptible to errors in transfer as affords opportunities to transmit visible actions and less visible actions". The authors argue that "high environmental uncertainty" heightens imitation learning as "firm's strategists use social comparison to select and evaluate their own actions". They expect imitation to occur less in Canada because there is not an intense competitive environment. Therefore the opportunities for learning may be minimised whereas "many hospitals in the United States publicize their investments in the transfer of patient safety best practices in an effort to demonstrate to regulators that efforts are being made in this arena".

(2) Characteristics of the hospitals that undertake knowledge transition knowledge has "tacit elements" that are not easily copied. "The exclusion or omission of routines that are less visible may have severe consequences for the success of a transfer because they may be just as, or more, crucial to the success of a safety related initiative as the routines that can be observed at arms length". So, for example, while it is more likely that an institution will copy a broad reporting system, they may well miss important changes in work processes and system design that require behaviour change on the part of providers.

(3) Characteristics of knowledge targeted for transfer

Transferring knowledge within organisations may be no less difficult. "Organizational practices can be identified and transferred by top management teams and dedicated staff, while unit-based practices require developing local expertise and changing unit work practices that are firmly embedded in the unit". But "although intrafirm transfers of knowledge are often laborious, time consuming and difficult, current conceptions continue to treat them as costless and instantaneous".

(4) Learning units' characteristics and interorganisational knowledge transfer

An important characteristic in knowledge transfer is the ability of the unit to change or its "absorptive capacity". This term describes a learning unit's ability to recognise the value of external information, assimilate it, and apply it. "One way that hospitals build absorptive
Knowledge transfer through imitation carries risks because 
capacity ... is by dedicating human resources to developing patient safety efforts". These include providing staff with opportunities to enhance transfer experience through meetings and conferences and rotating personnel to affect the transfer and exchange of tacit and explicit knowledge and to enhance their understanding the knowledge needs of their organisations where knowledge resides in them

(5) Transfer process characteristics and knowledge retention

Five retention repositories for knowledge in organisations exist:

- individual members;

- roles and organizational structures;

- standard operating procedures and practices;

- internal culture; and

- physical structure of the workplace.

"The number of repositories touched by patient safety best practice initiatives and the retention, or sustained use, of new knowledge about patient safety in a hospital are directly related".

The authors conclude: "Beyond the need to understand the technological components of patient safety improvement efforts, hospital managers need to understand the human, institutional, and structural factors associated with knowledge transfer and knowledge management relating to patient safety initiatives".

$\Delta$ Blair Berta W, Baker R. Factors that impact the transfer and retention of best practices for reducing error in hospitals. Health Care Manage Rev 2004;29:90-7

Learning from handovers in other industries to improve patient safety Like the paper by Walshe and Shortell, this also seeks to learn lessons for health care from high risk industries. It is concerned with handovers between staff, which are referred to as "handoffs". The objective in any handoff is the "accurate transfer of information about a patient's state and care plan". Handoffs are "a recognized point of vulnerability and their role is crucial". "Research in space shuttle mission control has identified some of the potential costs of failing to be told, forgetting or misunderstanding information communicated during a shift change handoff'". The authors list seven:

- having an incorrect or incomplete model of the system's state;

- being unaware of significant data or events;

- being unprepared to deal with impacts from previous events;

- failing to anticipate future events;

- lacking knowledge that is necessary to perform tasks;

- dropping or reworking activities that are in progress or that the team has agreed to do; and

- creating an unwarranted shift in goals, decisions, priorities, or plans.

"Therefore, in order to ensure system robustness during handoffs, effective communication and coordination strategies are needed".

To learn lessons for acute care, the authors observed handover procedures during personnel changes in space shuttle mission control, nuclear power generation, railroad dispatching, and ambulance dispatching and found strategies which they believe improve the efficiency and effectiveness of the procedure:

- In space shuttle mission control, the outgoing person wrote a one-paragraph summary of the shift in a log in preparation for the verbal handoff.

- The log feeds into an overview of the system and the incoming person assessed the current status of the monitored system ... made easier by "at a glance" overview displays.

- The incoming person scanned historical data immediately.

- The incoming person was expected to review automatically captured changes to sensor-derived data.

The main difference between handovers in acute care and others is the lack of technology to aid the transfer of information. "For instance, health care personnel do not have at a glance overview status and historical displays, and therefore have to convey more information during their updates".

The authors suggest that one way of improving handover in acute care would be for an outgoing nurse to verbally summarise information about each of the patients under his or her responsibility onto an audio tape. Everyone could listen to the tape "but only the nurse assigned responsibility for each individual patient takes handwritten notes while listening". However, as the authors note, this would not allow questioning from the incoming person, although the tapes could be listened to before the outgoing person leaves and an opportunity for "an explicit face-to-face check out" implemented. The authors suggest time could be protected during this handover to ensure there were no interruptions. Furthermore, they suggest that, if reports could be recorded digitally, they could provide an overview display system for everyone on the ward.

The technological investment required may be prohibitive and the culture of working would have to change accordingly but, say the authors, "an understanding of the state of the art in how handoffs are conducted in settings with high consequences for failure can be used to jumpstart endeavors to design handoffs in health care".

$\Delta$ Patterson $E$, Roth $E$, Woods D, et al. Handoff strategies in settings with high consequences for failure: lessons for health care operations. Int I Qual Health Care 2004; 16:125-32

Bringing together managerial and clinical approaches to improvement "In order to raise interest and foster commitment among staff, problems need to be locally identified, whereas the assessment of the importance of the problems for the whole organization calls for an overall view, requiring a central decision". While individual efforts focus on particular problems, organisations take a more comprehensive view of systems but do not necessarily involve "front line efforts". While "bottom up" approaches have much more effect on staff attitudes and behaviour than does the top down approach, "the involvement of organizational leadership is also critical to successes".

This led the researchers to pose a series of questions:

- Does it make a difference if problems are identified locally or centrally?

- Do clinical teams define and rate problems differently from managers?

- Do problems vary between organizational units and clinical specialties - are they specific or generic?

- What does the "epidemiology" of quality problems look like?

To help answer these problems the researchers undertook a study at Huddinge University Hospital (one of two teaching hospitals closely tied to the Karolinska Institute in Stockholm) where the management decided to introduce process management. The authors acted as facilitators in the project. All of the clinical directors at the hospitals identified two healthcare processes and appointed a senior physician as "process owner" and also formed and led a management team consisting of the heads of concerned departments. The process owners assembled teams of doctors, nurses, secretaries, and other professionals.

There were four stages to the project: (1) brainstorming to identify problems, (2) prioritisation and specification, (3) sample indicator measurement, and (4) management selection [of problems to take forward].

In the first stage team members recorded on sticky notes problems they knew or believed patients faced, based on their experience from daily contacts with patients. Team members then organized the many sticky notes (often 100+) according to where in the process each problem belonged. Next, team members voted to prioritise between problem groups and developed "problem statements" to specify the top six problems. In the second stage they sorted the notes into the area of the patient pathway to which they felt the problem belonged and in the third stage, where management became involved, the groups selected ways they felt improvements could be demonstrated. In the fourth stage the researchers presented the analysis and asked management to select projects to resolve key problems.

The main finding of the research is that the different stages led to new ways of formulating problems. In the first stage a small proportion of problems were categorised as falling within "waiting times" yet, by the third stage, "there was a clear shift in the proportions" and "waiting times emerged as the leading category".

The researchers suggest there are three reasons for the change:

- The criteria for selecting problems may have influenced the shift. Although waiting time problems were not most frequent after initial brainstorming, they may have fit better the criteria for selecting problems to work on later. 
- Waiting times may be particularly easy to define and measure.

- Waiting times are a political priority and important in the minds of managers and clinicians.

The researchers also accept that "the methodology for refining problems could have turned information issues and poor procedures into waiting time problems". They argue that the shift is positive and represents a recognition that, in presenting analysis in different ways, going through various stages - from personal experience to a systemic analysis - a route from frontline concerns to managerial problems can be ploughed and joined in a framework that relates different perspectives.

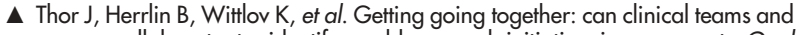
managers collaborate to identify problems and initiative improvement. Qual Manage Health Care 2004;13:120-42.

Reforming health systems Simon Stevens has been an advisor on health policy to the UK Government since 1997. He initially advised the Secretary of State and then became the Prime Minister's key aide on health. He announced he would leave in May 2004 and in an article for Health Affairs explains the multidimensional reforms set in place during his time.

Raising taxes to generate increases in funding has also raised the stakes because "if the extra investment fails to deliver more consumer responsive health care, the British people will probably conclude that it is the NHS model itself that is the problem". Given this, "policymakers' attention has accordingly switched from financing to supply-side questions: how best to expand output, improve quality, and increase responsiveness while avoiding cost inflation". It has involved 12 reform strategies in the following three dimensions:

- Support for providers.

- Hierarchical challenge.

- Localist challenge.

\section{(1) Support for providers}

Strategy 1: increasing the supply of health professionals, boosted by a $55 \%$ increase in medical school intake and other incentives

Strategy 2: modernising infrastructure: public hospitals are being rebuilt under partnership with the private sector and an IT investment programme is underway.

Strategy 3: supported learning and improvement: the NHS has established a Modernisation Agency which is responsible for horizontal spread of reengineering and service redesign techniques. It has also taken steps to establish a corporate university.

(2) Hierarchical challenge

Healthcare providers, as well as being altruistic and principled, can also occasionally be inefficient, variable in quality, selfinterested, and unresponsive to patients' preferences.

Strategy 4: national standards and targets.

Strategy 5: inspection and regulation: NHS hospitals are not subject to independent inspection, "doctors are subject to mandatory relicensing every five years, and a National Clinical Assessment Authority now assesses NHS doctors whose performance gives cause for concern".

Strategy 6: published performance information.

Strategy 7: direct intervention: under a system of "earned autonomy", providers who have high ratings "gain small financial bonuses but win much greater operational freedom".

(3) Localist challenge

Stevens says that pressure on providers should come not only from regulators or government "but instead from market incentives or local democratic accountability".

Strategy 8: active purchasing: the Government has devolved 75\% of NHS funding directly to local NHS primary care trusts, "capitated single payers" who purchase care for their local populations.

Strategy 9: patient choice puts an emphasis on choice of providers for patients, meaning they will be able to choose any provider who offers procedures at the new NHS tariff rate- "be they public, private or not-for-profit".

Strategy 10: aligned provider incentives: the NHS has created national tariffs for different DRGs. "By 2008 this national tariff system will provide sharper incentives for organizations while constraining hospitals pricing power and underpinning patient choice and more diverse supply".
Strategy 11: new entrants and plural supply: "Government is now stimulating a more mixed economy on the supply side to expand capacity, enhance contestability and offer choice. Freestanding surgical centres run by internal private operators under contract to the NHS are a first step. Private diagnostics and primary care 'out of hours' services are next."

Strategy 12: local democratic accountability: for those services with inherently "low contestability, choice and quasi-markets are unlikely to be adequate drivers of improvement"; pressure from local accountability will force responsiveness instead.

Stevens concludes that "the past five years have arguably seen three tectonic shifts in the post-war health policy settlement: an end to the bipartisan political consensus on the role of tax funding; a new bipartisan consensus on the value of a more mixed supply side; and increased challenge to the medical profession's power. In part this is because of an increasing appreciation of the fact that health care improvement requires a source of tension to overcome the inertia inherent in all human systems."

A Stevens S. Reform strategies for the English NHS. Health Aff 2004;21:37-44.

Influence of reward strategies on reform $>$ A paper in the Journal of Health Service Research \& Policy examines the difference in activity between English consultants. Variation was explored using Hospital Episode Statistics (HES) for England, linked with workforce data on consultant characteristics in given surgical specialties (general surgery, urology, trauma and orthopaedics, ENT and ophthalmology). HES data in 1998/99 were analysed and repeated for the year 1999/2000. Each observation in the extract from HES represented one finished consultant episode (FCE), defined as "a period of health care under one consultant in one hospital provider". "If consultants were employed on a part-time basis or honorary contract, their activity was inflated to a full-time equivalent rate based on a number of sessions they were contracted to provide to the $\mathrm{NHS}^{\prime \prime}$.

The results showed considerable variation between consultations with the top $25 \%$ of consultants having activity rates $60-85 \%$ higher than the bottom $25 \%$ with part-time contract holders being more productive that full-time colleagues. The authors consider that there are a number of potential reasons for the differences, but without further research they can only discussed speculatively.

"The HES dataset identifies the activity of a consultant's team by assigning it to one consultant. Data are not collected centrally on the size of teams working to consultants, so it is not possible to account for team size in the model. It may also be that full-time consultants are more likely to undertake responsibility other than inpatient care, such as administrative and managerial roles, and teaching and training. Alternatively, those who undertake substantial practice in the private sector may perhaps develop a faster practice style, or may focus on inpatient activity, conducting relatively little outpatient activity, which is not currently included in HES."

A new contract for consultants has been agreed in 2004. The authors consider that "the new system of mandatory job planning and appraisal will require improved systems of monitoring and management. Similarly, any additional incentive schemes that develop will also require good measurement of consultant activity in order to reward those who achieve the most. Providing easily interpretable graphical data to NHS hospitals represents a first step in the process of managing the variation."

The authors question the capacity of management to use information-even that which already exists. "The majority of managers have, for decades, failed to monitor the existing consultant contract, ignoring data such as HES, and the existence of well-chronicled large practice variations. Change in management attitudes and practice must be induced and sustained and use of activity data is an essential first step in this process."

$\Delta$ Bloor K, Maynard A, Freemantle N. Variation in activity rates of consultant surgeons and the influence of reward structures in the English NHS. J Health Serv Res Policy 2004;9:76-84.

Using incentives to enhance quality in primary care - This paper describes a major experiment in the UK with explicit quality incentives in primary care [under a new contract for GPs] which marks one of the most ambitious attempts to "incentivize quality to date".

GPs have traditionally been offered a wide range of direct financial incentives via a national contract that offers a mix of remuneration methods including fee-for-service (about 15\% of GP 
income), capitation (40\%), salary (30\%), and capital and information technology (15\%). They are thus used to working within an "incentivised environment" and, although there is little evidence on the impact of incentive mechanisms, evidence does suggest that GPs do respond as predicted to material incentives. For example, under GP fundholding (an experiment from 1991-8), practices were given annual budgets with which to purchase non-emergency secondary care and pharmaceuticals for their patients. In response to the budgetary regime, fundholders reduced inpatient procedures and secured shorter waiting times for their patients. A quality improvement scheme in southern England was tested from 1998 to 2000 Nearly $80 \%$ of local GPs enrolled in a programme that required them to meet challenging chronic disease management targets across 13 conditions. GPs were offered a financial incentive (about $5 \%$ of earnings) to adhere to standards. An evaluation found material improvements.

This scheme "influenced a major change to primary care incentives that was embodied in an entirely new GP contract, introduced in April 2004". Central to the new contract is a system of quality incentives. About $£ 1.3$ billion, around $18 \%$ of GP income, will be distributed annually on the basis of quality measures. The new contract uses 146 indicators of quality across seven areas of practice. In each area a certain number of quality "points" are available, up to a maximum of 1050. About half of these are for clinical quality. Other areas include practice organisation (184 points) and patient experience (100 points). The scheme will reward practices (rather than individual physicians), so it is likely to encourage teamwork and peer review and "there is a commitment to reviewing and updating the incentive scheme".

"Notwithstanding [its] apparent strengths, there are some potential risks: it is a complex scheme and GPs may not understand its full implications, responding as intended and important areas not covered by the scheme may be downgraded". It will be "important to check whether the scheme adversely affects some of the 'soffer' quality attributes of primary care that are not directly rewarded, such as continuity and advocacy or collaborative actions with other public services. Will such a heavy reliance on the explicit use of incentives make GPs less willing to respond in the best interests of patients when not directly rewarded?"

The authors conclude that an early evaluation of the scheme is clearly a high priority but, although it has risks, many of its design features accord well with the principles of incentive design advocated by economists.

A Smith P, York N. Quality incentives: the case of UK general practitioners. Health Aff 2004;23:112-8.

What is patient centred care? A paper in Health Expectations begins by noting that patient centred care "covers a range of activities from patient involvement in individual care to public involvement in health policy discussions". It can be understood as a continuum of activities or, more loosely, as a concept that has yet to be defined in practice. To better understand how it will develop, the researchers interviewed "intermediate level stakeholders such as health service managers, educationalists, professional leaders and officers of patient bodies" to see how they understand the concept and are seeking to take it forward.

From 47 interviews the researchers found a "typology of meanings" that reflected the interviewees' backgrounds and perspectives. "Several respondents talked about the notion of placing the patient at the centre of the care process, but what exactly this meant was not well articulated". Some highlighted the importance of healthcare relationships as "partnerships", encompassing the need for "shared information and decision-making". "Those drawn from health professional groups ... reflected the need for health professionals to inform patients and engender trust" $-a$ more professionally led notion. "In contrast, respondents from usergroups often described patient-centred care in the context of a social or whole person model of health".

In examining "the use of the term made by policymakers", the authors see "the general thrust of policies [focusing] on systems and organizations rather than on what actually happens at the level of individuals". "What is missing is a clear recognition among policymakers, managers and health professionals ... of the full spectrum of potential activities which constitute patient centred care, from public involvement to individual interactions".
The authors find this "paradoxical", given that only a minority of patients will ever be involved in the planning and organization of services but virtually all will come into contract with health services at an individual level. They wonder whether policymakers see this individual end as a clinical matter more properly the concern of clinicians and health professionals than of managers. In any case, the findings of this study "highlight a gulf between the increasing literature on shared decision making and our respondents' understandings [and] until a more complete understanding of patient centred care is accepted, the further development of patient centred care mediated by these professionals is unlikely".

A Gillespie R, Florin D, Gillam $S$. How is patient-centred care understood by the clinical, managerial and lay stakeholders responsible for promoting this agenda? Health Expectations 2004;7:142-8.

What models of relationships with health professionals do different patients prefer? In the last two decades the term "patient autonomy" has appeared more and more frequently in the medical literature. A textword search in PubMed by a team of Dutch researchers provided 41 citations for 1980 and 324 for 2000. This prompted them to ask: what does autonomy mean and what kind of relationship with professionals does such a term imply?

"In the medical literature authors generally seem to imply the liberal individualist concept of autonomy ... in which autonomous patients are choosers who act intentionally and without controlling influences that determine their actions. According to this interpretation of autonomy, the goal for an autonomous person is to decide on his or her own, without undue manipulation by others." However, from a descriptive literature of how patients might make decisions, ideas have emerged that many patients do not have a strong preference for autonomy in the liberal individualist sense.

The researchers wished to "assess whether aspects of ethical theories other than liberal individualism can be distinguished in medical practice". They set out to explore patients' and surgeons' ideals of autonomy, beginning with six moral concepts of patient autonomy. Factor analysis was carried out on 55 statements, answered on a 5-point statement (ranging from fully disagree to fully agree) that aimed to characterise what constituted a good relationship, based on individual norms and values.

Two extremes of autonomy emerged:

- Doctor knows best (statements included: "if doctors and patients cannot agree on which treatment is best, the doctor should make the treatment decision").

- Patients should decide (the patient must choose between the various treatments: "if a patient chooses a treatment with more health risks, the doctors should respect this treatment decision").

Two other factors which sat between the classic polar positions of the autonomy debate were also identified:

- Right to non-participation ("if the patient does not want to receive information about risks, the doctor should respect this"; "patients who become afraid when thinking about the treatment decision should be left in peace by the doctor").

- Obligatory risk information (the patient has to be informed of all the risks).

"By incorporating aspects from various ethical theories, a subtler picture of the physician-patient role emerges than is generally suggested by the medical literature. Two of the four factors reflect the well-known distinction between physician-centred and patientcentred decision making. The third is a subtle but important aspect of the patient-physician relationship, namely the right of the patient to decide whether to delegate the decision back to the physician. The fourth reflects the right and the duty of patients to be made aware of relevant risks."

A Stiggelbout A, Molewijk A, Otten W, et al. Ideals of patient autonomy in clinical decision making: a study on the development of a scale to assess patients' and physicians' views. J Med Ethics 2004;30:268-74.

T Smith

Senior Policy Analyst, Health Policy and Economic Research Unit, British Medical Association, London WC1H 9JR, UK; tsmith@bma.org.uk 